Щ्)FRANÇAISE

$>\mathrm{DE}$

땓 PÉDAGOGIE

\section{Revue française de pédagogie}

Recherches en éducation

156 | juillet-septembre 2006

Les espaces locaux d'interdépendance entre

établissements : une comparaison européenne

\title{
Compétition et fonctionnement des établissements scolaires : les enseignements ) d'une enquête européenne
}

How schools work and compete: learning from a European survey

Competición y funcionamiento de los establecimientos escolares: las lecciones de una encuesta europea

Wettkampf und Betrieb der Schulanstalten: die Ergebnisse einer europäischen Untersuchung

\section{Agnès Van Zanten}

\section{CpenEdition}

Journals

Édition électronique

URL : http://journals.openedition.org/rfp/263

DOI : $10.4000 /$ rfp. 263

ISSN : 2105-2913

Éditeur

ENS Éditions

Édition imprimée

Date de publication : 1 juillet 2006

Pagination : 9-17

ISBN : 978-2-7342-1060-3

ISSN : 0556-7807

Référence électronique

Agnès Van Zanten, « Compétition et fonctionnement des établissements scolaires : les

enseignements ) d'une enquête européenne », Revue française de pédagogie [En ligne], 156 | juilletseptembre 2006, mis en ligne le 27 septembre 2010, consulté le 10 décembre 2020. URL : http:// journals.openedition.org/rfp/263; DOI : https://doi.org/10.4000/rfp.263 


\title{
Compétition et fonctionnement des établissements scolaires: les enseignements d'une enquête européenne
}

\author{
Agnès van Zanten
}

Cet article s'intéresse aux effets sur le fonctionnement des établissements de la compétition autour de la ressource principale que représentent les élèves, notamment les "bons "élèves, et ce à partir de données recueillies dans six espaces locaux de cinq pays européens. II examine en premier lieu les effets de la compétition sur différents domaines d'activité des établissements : le recrutement des élèves, l'offre d'options, les stratégies de promotion, l'organisation des classes, l'aide aux élèves en difficulté et la discipline. II analyse ensuite les variations entre établissements dans les réponses à la compétition en fonction du degré de concurrence et de leur position hiérarchique dans les contextes locaux considérés. Les conclusions mettent l'accent sur les multiples effets de la compétition, malgré les variations locales et nationales, et sur la nécessité de tenir compte de leur influence, à côté de celle des politiques éducatives nationales et locales et de celle des dynamiques internes, dans l'étude de l'activité des établissements. Ceci s'avère d'autant plus important que beaucoup de ces effets apparaissent négatifs car ils tendent à renforcer les ségrégations et les inégalités entre établissements et à l'intérieur de ces derniers.

Descripteurs (TEE) : compétition, Communauté européenne efficacité scolaire, établissements d'enseignement, gestion d'établissement scolaire, ségrégation.

es analyses sur le fonctionnement des établissements scolaires adoptent le plus souvent l'une des deux perspectives suivantes: soit elles se focalisent sur les effets de différentes politiques nationales ou locales, notamment celles visant à doter les établissements de plus d'autonomie, soit elles se centrent sur les jeux internes entre les professionnels de l'éducation et, plus particulièrement, entre les enseignants et entre ces derniers et les chefs d'établissements. Ces deux perspectives permet- tent de saisir des aspects importants de l'orientation générale et du quotidien des établissements, mais elles ne permettent pas une vue compréhensive de leur activité car elles évacuent le plus souvent le fait que les établissements sont des organisations encastrées dans des environnements sociaux et institutionnels avec lesquels ils interagissent et qui affectent leur fonctionnement (Meyer \& Rowan, 1978). Plusieurs dynamiques sont à l'œuvre dans cette relation entre les établissements et leur environnement avec 
notamment, d'un côté, des relations de collaboration donnant lieu à des régulations de type communautaire ou réticulaire et, de l'autre, des relations de compétition donnant lieu à des régulations de type marchand, au sens d'une prédominance du jeu de l'offre et de la demande (Maroy \& Dupriez, 2000).

Dans cet article, et dans la recherche comparative européenne qui constitue sa base empirique, nous nous sommes davantage intéressée à ce dernier type de phénomène au travers de la notion d'« interdépendances compétitives ». Cette notion désigne le fait que le fonctionnement des établissements est affecté par les pratiques des établissements situés dans le même espace ou dans des espaces voisins, avec qui ils sont en concurrence pour des ressources convoitées par chacun, notamment les élèves (Delvaux \& van Zanten, 2004 ; cf. supra, l'introduction). L'ampleur et la nature de ces interdépendances compétitives dépendent des règles de jeu établies par la régulation politique centrale ou intermédiaire, mais aussi de processus locaux, de sorte que l'on peut avoir affaire à des interdépendances compétitives entre écoles sans nécessairement se trouver dans un système institutionnel qui promeut ces modes de régulation. Toutefois, si l'hypothèse de départ était que la compétition apparaîtrait comme une dimension structurante à part entière de l'activité des établissements, les conclusions des recherches de terrain invitent à nuancer cette hypothèse sur deux points. D'une part, toutes les dimensions de l'activité des établissements ne sont pas concernées au même degré par la compétition et, d'autre part, tous les établissements ne sont pas affectés dans les mêmes proportions et de la même façon par celle-ci. C'est ce que nous allons essayer de montrer ici en insistant sur les facteurs qui expliquent les différences entre les cinq pays étudiés (Angleterre, Belgique, France, Hongrie, Portugal) et entre les établissements.

\section{L'INFLUENCE DE LA COMPÉTITION SUR LES DIFFÉRENTS DOMAINES D'ACTION DES ÉTABLISSEMENTS}

Pour observer de façon relativement détaillée les effets des interdépendances compétitives sur le fonctionnement des établissements, il est utile, dans un premier temps, d'examiner séparément différentes dimensions de leur activité. Dans la recherche, il a été possible d'isoler six domaines présents dans le fonctionnement de tous les établissements, et affec- tés à des degrés divers par la compétition. Dans les analyses qui suivent, nous distinguons trois domaines d'action orientés prioritairement vers l'environnement externe (le recrutement, l'offre d'options et les activités de promotion des établissements), dont on peut supposer qu'ils sont les plus sensibles aux phénomènes de concurrence. Nous évoquons aussi, cependant, trois domaines d'action dont les dynamiques répondent principalement à des pressions internes (l'organisation des classes, les dispositifs de soutien aux élèves en difficulté et la discipline) mais qui peuvent être aussi affectés par la compétition. Nous y signalons quelques différences entre les contextes nationaux et locaux, qui renvoient à des facteurs politiques, organisationnels et culturels.

\section{Les domaines d'action orientés vers l'extérieur}

Un des domaines d'action les plus importants pour les établissements est celui du recrutement des élèves car le nombre et la "qualité » de ces derniers commandent en grande partie l'obtention des ressources et influent fortement sur les conditions de travail et le prestige des professionnels de l'éducation. Par ailleurs, "l'effet public » pèse lourdement tant sur les résultats des établissements que sur leur attractivité. Toutefois, la capacité d'action des établissements dans ce domaine est limitée par au moins trois facteurs : le nombre de places, le système de régulation des flux d'élèves et les stratégies des familles. La comparaison internationale montre que les établissements belges et hongrois sont les plus portés et les plus aptes à développer des actions en matière de recrutement en raison de l'organisation politique et administrative locale et nationale (libre choix de l'école et financement des établissements par " tête d'élève "), mais aussi, surtout à Budapest, d'une baisse démographique très importante qui fait craindre aux établissements la perte de postes et, à terme, la fermeture. Mais si tous les établissements sont engagés dans une compétition de «premier ordre » pour avoir assez d'élèves, ils sont aussi engagés dans une compétition de "second ordre". Celle-ci porte d'abord sur les meilleurs élèves au plan académique, mais peut aussi se tourner vers la sélection d'élèves " motivés " et " calmes " (Gewirtz et al., 1995). Dans l'espace local étudié à Londres et dans les deux espaces locaux français, on observe surtout une compétition de "second ordre ». Celle-ci reste néanmoins limitée face à la régulation du secteur public par un système de banding (répartition des élèves entre les établissements par un système de quotas en fonction de leur niveau académique) à Londres et par la sectorisation des établissements en France, celle-ci étant 
néanmoins appliquée de façon plus stricte en région parisienne qu'à Lille. Signalons pour conclure que c'est au Portugal que le recrutement semble le moins affecté par la compétition à la fois en raison de l'existence d'une carte scolaire et d'une demande moins forte en faveur du choix de l'école de la part des parents et de l'importance d'une éthique du service public parmi les professionnels de l'éducation.

L'offre d'options est un deuxième domaine d'action orienté vers l'extérieur et étroitement lié au recrutement. Dans ce domaine, on observe de nombreuses tentatives pour mieux soutenir la concurrence d'autres établissements. II est en effet apparu dans la recherche que, dans la plupart des contextes étudiés, les établissements demandent de nouvelles options moins dans une optique d'enrichissement et de diversification de l'offre curriculaire en lien avec des finalités de développement intellectuel ou d'insertion des élèves dans le marché du travail que dans une optique de meilleur positionnement vis-à-vis des élèves de « bon » niveau scolaire et social qu'ils cherchent à attirer. Cependant, la volonté et la capacité des établissements à agir dans ce domaine dépendent de la palette d'options qu'autorisent les autorités éducatives locales ou nationales et du degré de contrôle de leur distribution entre les établissements. Ce contrôle s'est avéré assez fort dans tous les contextes nationaux, sauf à Londres et à Budapest. Dans le premier cas, les établissements, comme dans l'ensemble de l'Angleterre, sont poussés par les autorités à développer une offre spécialisée leur permettant de s'adresser à des niches particulières au sein de la population et, surtout, d'obtenir des ressources supplémentaires. Dans le second, ce sont les établissements qui se mobilisent pour créer des classes «spéciales 》 (bilingues, à mathématiques renforcées ou proposant des pédagogies spécifiques pour les élèves en difficulté) car la réglementation en vigueur émanant de la municipalité n'exige que le maintien d'au moins une classe « normale » accueillant le tout-venant des élèves du district. Des stratégies similaires, mais plus contrôlées par les autorités, ont été observées en Belgique ou en France. Au Portugal, les établissements ne semblent pas développer des stratégies distinctives en matière d'offre par manque d'autonomie, mais aussi par manque d'incitation dans ce domaine. Une certaine spécialisation existe néanmoins au bas de l'échelle de prestige dans la mesure où seuls certains établissements ont des classes avec des "curricula alternatifs " à dominante professionnelle en direction des élèves pour lesquels la poursuite des études n'est pas envisagée.
Un dernier domaine d'action des établissements orienté vers l'extérieur concerne leurs stratégies de promotion. Celles-ci sont très liées aux deux dimensions traitées précédemment dans la mesure où leur objectif prioritaire est d'attirer des élèves et où elles sont le principal moyen l'affichage d'une offre attrayante pour le type de public visé. Ces activités sont à la fois plus légitimes et plus nombreuses dans les contextes où les établissements sont autorisés à afficher des profils différents et à choisir leurs élèves, ce qui était le cas dans notre étude notamment à Londres, à Charleroi et à Budapest, où les établissements distribuent des plaquettes, passent de petites annonces, ont des sites Internet et organisent diverses activités pour se faire connaître. Elles sont apparues particulièrement importantes dans le contexte britannique car les établissements, dans le but d'attirer des élèves de meilleur niveau mais aussi de nouveaux financements en provenance de l'État et des partenaires privés, soignent beaucoup leur image par des contacts avec les écoles primaires, le travail en commun avec diverses organisations non éducatives locales, la mise en place de diverses manifestations en direction de la communauté et des prestations dans les médias. En revanche, ces activités sont plus discrètes - mais non inexistantes - dans les deux contextes locaux français dans la mesure où le mode de régulation en vigueur concernant le secteur public limite fortement la différenciation des établissements et leur choix par les familles. Elles sont néanmoins importantes dans certains établissements privés. Elles paraissent peu présentes dans les établissements portugais à la fois en raison d'une législation encore fortement orientée vers la standardisation de l'offre éducative et d'une moindre demande sociale de différenciation.

\section{Les domaines d'action orientés vers l'intérieur}

Parmi les domaines d'activité des établissements qui répondent davantage à des pressions internes, un des plus importants concerne l'organisation des classes. En effet, dans tous les systèmes locaux étudiés, et notamment dans les établissements caractérisés par une hétérogénéité importante des niveaux des élèves, leur regroupement en fonction de caractéristiques académiques (mais qui recoupent le plus souvent des caractéristiques sociales, sexuelles et ethniques) constitue un enjeu interne de taille car il conditionne directement le travail des enseignants et, indirectement, le fonctionnement pédagogique et disciplinaire de l'ensemble de l'établissement. Mais, en outre, dans plusieurs de ces systèmes, cette organisation 
interne joue aussi un rôle de "signal » en direction des parents des classes moyennes et supérieures qui craignent les effets de l'hétérogénéité sur les progrès scolaires mais aussi sur le développement personnel et la socialisation de leur progéniture (van Zanten, 2002). Ce phénomène est apparu très important dans notre étude dans le cas de Budapest pour des raisons financières car, comme nous l'indiquons plus bas, les établissements tirent profit de la présence d'élèves défavorisés qu'ils regroupent toutefois dans des classes spécifiques pour ne pas nuire à la scolarité des autres. II est aussi relativement important en France où il compense la faible capacité des établissements à proposer une offre attractive d'options pour retenir et attirer les « bons » élèves et ce malgré l'interdiction officielle de constituer des classes de niveau. II revêt en revanche moins d'importance dans les contextes où la différenciation entre les établissements est plus forte, comme en Angleterre et en Belgique, ou dans ceux où le public d'élèves à l'intérieur des établissements est moins hétérogène, comme dans la zone étudiée à Lisbonne.

Les activités de soutien aux élèves en difficulté constituent un autre domaine d'action dont le fonctionnement est plus orienté encore par des facteurs internes, notamment les caractéristiques des élèves (qui découlent cependant des processus externes faisant souvent intervenir la compétition entre établissements) et les dispositions éthiques des professionnels de l'éducation qui se développent en lien avec ces caractéristiques (van Zanten, 2001). Les dispositifs d'aide peuvent néanmoins être utilisés pour obtenir des ressources financières additionnelles. Ceci a été observé principalement à Budapest où les écoles spécialisées dans le traitement des publics en difficulté peuvent obtenir des subventions spécifiques (par exemple une allocation normative, dite ethnique) et où une partie du corps enseignant a suivi une formation de pédagogue spécialisé et intervient parallèlement à une grande diversité de personnels parascolaires (logopèdes, kinésithérapeutes, psychologues, psychiatres...). Ces activités peuvent aussi servir à rassurer les parents des "bons " élèves concernant le risque de " contamination " par des élèves plus faibles. Très souvent, cependant, c'est plutôt l'absence de telles activités qui est révélatrice d'une volonté de montrer une meilleure image dans un environnement concurrentiel, les établissements mobilisés dans ce sens choisissant de ne pas mettre en place de dispositifs de ce type pour éviter d'être stigmatisés comme étant des établissements en difficulté. Ces orientations ont été observées dans certains établissements français, belges, anglais et portugais, qui préfèrent exclure les élèves en difficulté en les orientant vers des filières techniques et professionnelles présentes dans d'autres établissements. Leur ampleur varie néanmoins en fonction du degré de contrôle et de condamnation morale de ces pratiques, qui apparaît plus fort en France et en Portugal et plus faible en Belgique et surtout en Angleterre où les établissements sont plus tiraillés qu'ailleurs entre la recherche d'équité et les demandes d'efficacité et d'innovation dont ils font l'objet de la part du gouvernement.

Enfin, un dernier domaine d'action est celui de la discipline. II s'agit à nouveau d'un domaine dont le fonctionnement est davantage conditionné par des régulations politiques et par des processus internes que par les interdépendances compétitives entre établissements et dont l'importance s'accroît au fur et à mesure que l'on se déplace des établissements situés en haut de l'échelle vers ceux situés en bas de celle-ci. Toutefois, des établissements favorisés peuvent avoir intérêt à afficher des pratiques strictes dès lors qu'ils cherchent à attirer une fraction " conservatrice " de parents, très attentifs à la bonne tenue des élèves. Cela était clairement le cas de certains établissements privés en France et du réseau libre en Belgique et de certaines écoles pour filles en Angleterre. Mais ce sont plus souvent encore des établissements jouissant d'une moins bonne réputation qui s'orientent dans ce sens afin de "rassurer »les parents des classes moyennes et supérieures. Dans tous les pays, ces établissements ont recours soit à des pratiques répressives, incluant les exclusions définitives, soit à des formes diverses de négociation et de contractualisation relevant de nouvelles formes de gestion post-bureaucratiques dans le système d'enseignement (Verhoeven, 1999), soit à des stratégies de «management des impressions " par l'occultation des problèmes. Ils peuvent aussi, comme en Hongrie et dans certains établissements belges, opter pour un traitement «thérapeutique » des problèmes de comportement des élèves par le biais notamment d'interventions d'ordre médical ou psychologique.

\section{L'INFLUENCE DE LA COMPÉTITION SELON LES ÉTABLISSEMENTS}

Si on peut donc conclure que la plupart des domaines d'activité des établissements, aussi bien ceux soumis à des pressions à dominante externe que, à un moindre degré, ceux soumis à des pressions à dominante interne, sont affectés par les dynamiques 
de compétition bien qu'inégalement et différemment suivant les contextes nationaux et locaux, il reste à explorer le poids de la variable "établissement". L'analyse détaillée des dynamiques locales dans les six zones étudiées, mais aussi du fonctionnement de quatorze établissements à l'intérieur de cellesci, montre en effet de grandes variations à l'intérieur d'un même espace dans les réponses aux interdépendances compétitives. L'organisation interne des établissements, notamment le degré de consensus entre le chef d'établissement, les enseignants et les parents, joue un rôle dans ces variations. Néanmoins, les deux facteurs qui semblent exercer l'influence la plus forte sont, d'une part, l'intensité de la compétition, qui pousse les établissements à développer des logiques d'action en réponse à celle-ci et, d'autre part, la position de l'établissement dans la hiérarchie locale, définie à la fois par ses résultats et par les caractéristiques de son public. En effet, selon que l'établissement se situe en haut ou en bas de l'échelle locale, cette position constitue son principal atout ou son principal handicap, pour faire face à la compétition. En croisant ces deux dimensions et en adoptant un point de vue idéaltypique, plutôt qu'en cherchant à rendre compte de façon fine de toutes les nuances, on peut alors faire état de quatre logiques dominantes. Deux d'entre elles, la logique de conquête et la logique de captation, correspondent à des situations de forte compétition et deux autres, la logique de rente et la logique d'adaptation, à l'absence ou au refus de la compétition.

\section{Des établissements pris dans le jeu de la compétition}

Comme nous l'avons suggéré dans la partie précédente, l'intensité de la compétition dépend d'un ensemble complexe de facteurs. Parmi ceux-ci, il y a d'abord ceux d'ordre politique et administratif (le degré de libre choix de l'école, les modes de financement et l'autonomie des établissements notamment). Les facteurs démographiques et sociaux (baisse de la population, caractéristiques sociales et degré d'hétérogénéité de celle-ci, processus de gentrification ou, au contraire, de paupérisation des quartiers) jouent cependant aussi un rôle important. Enfin, les caractéristiques de l'offre sont également déterminantes (nombre et accessibilité des établissements, degré de hiérarchisation et de différenciation entre eux) car il ne suffit pas d'avoir la possibilité légale de choisir ; il faut encore que le choix puisse se concrétiser et qu'il apparaisse désirable (Yair, 1996). À cela, il faut ajouter que si cette intensité est une dimension qui peut être objectivée par la mesure notamment des changements d'élèves entre établissements, elle fait l'objet d'une appréciation subjective de la part des professionnels de l'éducation, notamment des directeurs, généralement les plus attentifs aux interdépendances compétitives. Le degré de compétition est en effet évalué par ces derniers en fonction d'informations, les plus souvent très imparfaites, sur les flux d'élèves entre les établissements (Bagley et al. 1996). II est apprécié également suivant des postures éthiques quant au caractère bon ou mauvais de la compétition, qui varient selon les individus, mais dépendent aussi des contextes locaux et des cultures nationales (Ball \& van Zanten, 1998). II est enfin plus ou moins pris en compte selon que les directeurs conçoivent leur rôle comme tourné principalement vers les relations externes, soit comme impliquant prioritairement un leadership pédagogique interne (van Zanten, 1999).

Par ailleurs, la position de l'établissement détermine fortement son désir de s'engager dans la compétition, le parti qu'il peut en tirer et les stratégies qu'il développe. Confrontés à des situations ouvertes et instables quant à la distribution des élèves, les établissements dotés d'atouts importants, en fonction de leurs résultats et des caractéristiques de leur public, vont le plus souvent développer de logiques de "conquête" pour attirer des meilleurs élèves. Ceci notamment dès lors qu'il ne s'agit pas d'établissements "phares" mais d'établissements qui, bien que jouissant d'une bonne réputation, doivent se repositionner par rapport à ces bastions (Ballion, 1991). Ces établissements accordent beaucoup d'importance à tous les domaines d'activité dirigés vers l'extérieur, à savoir l'offre éducative, le recrutement et la promotion de l'établissement. L'offre est souvent renouvelée afin de proposer de nouvelles formes distinctives d'excellence, par exemple des classes européennes ou «bi-langues " dans les établissements français de Lille ou de la région parisienne ou des classes offrant des " parcours accélérés " pour les meilleurs élèves à Budapest. Cette offre est surtout dirigée vers les parents cadres ou managers du secteur privé qui sont à la fois plus mobiles et plus modernistes que les autres parents des classes moyennes et supérieures. Ces établissements, notamment les établissements que nous avons appelés "libres ", qui jouissent de plus de liberté dans ce domaine, mettent aussi en place de nouvelles stratégies de sélection des élèves, par exemple des tests d'évaluation du niveau académique à l'entrée, et développent des stratégies de promotion plus offensives que d'autres établissements car ils ont besoin de faire connaître et reconnaître leur offre, les qualités de leur public et leurs résultats. Ils 
tendent en revanche à accorder peu d'importance au soutien aux élèves en difficulté. Par ailleurs, du fait de l'importance accordée à la projection de l'établissement vers l'extérieur, les personnels de direction jouent un rôle central dans ce type d'établissements. Toutefois, la logique de conquête ne peut réussir que si certains groupes d'enseignants ou de parents soutiennent au moins tacitement leurs choix.

De leur côté, les établissements situés au bas de l'échelle des résultats et de prestige ou occupant une position moyenne, qui se trouvent encastrés dans des contextes locaux très compétitifs, sont eux aussi poussés à réagir, sous peine de devenir des établissements "ghettos". Ne disposant pas des mêmes ressources que les établissements situés en haut de l'échelle locale pour mettre en place des stratégies ambitieuses vis-à-vis de l'extérieur, ils sont dès lors contraints de développer de tactiques plus ou moins efficaces de captation des élèves. Certains établissements optent ainsi pour la création d'options moins prestigieuses que celles proposées dans les établissements les plus réputés, mais qui leur semblent encore susceptibles d'attirer des élèves de niveau bon ou moyen. C'est le cas de certains établissements belges de notre enquête par exemple qui proposent des options de sciences sociales ou des options artistiques plutôt que des langues anciennes. Quand l'éventail d'options est réduit, ces établissements se tournent plutôt vers la constitution de classes de niveau comme moyen de retenir les bons élèves, ceci étant particulièrement le cas en France et en Hongrie. Leur logique est en effet souvent une logique de diversification de l'offre interne, qui doit à la fois réussir à capter de bons élèves tout en permettant de faire face - soit de façon minimaliste, soit de façon plus ambitieuse - aux besoins des élèves en difficulté. Dans les contextes où, comme à Londres et à Charleroi, les établissements disposent d'une marge d'autonomie plus ou moins grande pour choisir leurs élèves, ils développent aussi des formes de sélection fondées sur des critères comportementaux, leur cible prioritaire étant des élèves faisant preuve de bonnes dispositions vis-à-vis des apprentissages et de l'école et soutenus à la maison. Ces établissements cherchent aussi à améliorer leur image en se focalisant sur le domaine de la discipline par le biais des stratégies d'occultation de problèmes ou de leur traitement répressif ou thérapeutique. Ces différentes tactiques s'avèrent efficaces dès lors qu'il existe un certain consensus entre la direction et les enseignants et qu'une fraction de parents des classes moyennes et supérieures choisit de «jouer le jeu » en restant loyaux à l'établissement local malgré ses imperfec- tions, quitte à compenser et contrôler son action (van Zanten, 2006). Toutefois, dans de nombreux cas, ces tactiques ne s'avèrent pas suffisantes pour limiter le pouvoir d'attraction des établissements « entrepreneurs " sur la population d'élèves " convenables» qu'ils cherchent à retenir ou à attirer.

\section{Des établissements hors-jeu}

La compétition peut néanmoins être faible au sein de certaines zones, ou pour certaines catégories d'établissements au sein de celles-ci, soit en raison de règles politiques et administratives interdisant ou limitant les choix des familles et des établissements, soit en raison de la stabilité de l'environnement social ou institutionnel. On n'observe pas dans ce cas de changements notables ni au sein de la population, ni à propos des établissements, qui soit occupent des places précises dans la hiérarchie locale, soit s'adressent à des " niches " particulières comme les établissements juifs orthodoxes étudiés dans le borough de Londres. Dans ce cas de figure, les établissements en haut de la hiérarchie peuvent maintenir des logiques d'action de « rentiers". Se trouvant à l'abri de la compétition, ils se contentent d'entretenir leur bonne réputation grâce à un maintien sans trop d'effort de leurs pratiques de sélection, puisqu'ils sont par définition très demandés. De ce fait, ils développent peu de stratégies de promotion, leur réputation bien établie se transmettant par ouïdire, et se montrent peu novateurs en matière d'une offre d'enseignement qui demeure très classique avec, notamment dans les établissements belges et français, un maintien des langues anciennes et un poids prépondérant des mathématiques comme moyen de sélection des meilleurs. Ceci s'explique entre autres par le fait que ces établissements, par opposition aux établissements " conquérants", ont moins pour cible les managers modernistes que les cadres et les professionnels plus attachés au maintien de l'enseignement à l'ancienne, même si la «tradition " continue à faire des adeptes parmi les nouvelles fractions des classes moyennes et supérieures (Edwards \& Whitty, 1997). À l'attachement à un curriculum classique s'ajoute souvent l'importance accordée au maintien d'une bonne discipline par l'application de règles strictes, ceci étant particulièrement vrai des établissements «libres ». Dans ces établissements, il existe souvent un accord profond entre tous les membres de la communauté éducative autour d'un idéal d'excellence et de rigueur dont la pertinence est légitimée par l'efficacité et l'attractivité de l'établissement. La recherche nous 
a néanmoins permis de constater qu'il existe des établissements, devenus au fil des années moins efficaces et moins attractifs, qui continuent à agir comme s'ils étaient en situation de monopole. Ces établissements se caractérisent généralement par la présence d'un chef d'établissement peu dynamique et d'enseignants âgés avec une longue carrière dans l'établissement.

À l'autre extrême de la hiérarchie locale, tant du point de vue des résultats que des caractéristiques du public, on trouve également des établissements qui se placent en position de «hors-jeu» par rapport à la compétition mais pour des raisons très différentes. II s'agit le plus souvent d'établissements qui, dans la plupart des contextes étudiés, ont été et sont encore gravement affectés par des interdépendances compétitives ayant renforcé la concentration en leur sein d'élèves en difficulté issus majoritairement de milieux défavorisés. Sauf dans les contextes comme celui de Londres, où tous les établissements sont poussés vers l'entrepreneuriat vis-à-vis de l'extérieur, ces établissements choisissent cependant de ne pas réagir à la compétition. Ceci reflète clairement leur manque de ressources car à une mauvaise image qu'il est quasiment impossible d'améliorer dans le court terme, s'ajoute souvent un manque de consensus interne lié à la mobilité des personnels et à la diversité des analyses concernant les causes des difficultés et les moyens d'y remédier. Tournant le dos aux dynamiques externes, les professionnels de ces établissements développent alors à l'interne une posture que l'on peut appeler d'« adaptation résignée ». Elle se caractérise par le développement d'éthiques et de pratiques professionnelles «humanitaires» qui visent davantage à maintenir un bon climat relationnel avec les élèves et à réduire leur souffrance en leur témoignant du respect et de la compassion qu'à encourager leurs progressions scolaires. L'activité de l'établissement est également focalisée sur les questions de discipline qui donnent lieu à une intense activité de négociation informelle et formelle avec les élèves. On y observe en fait un abandon des perspectives réflexives de développement intellectuel et des perspectives instrumentales de réussite des enfants en faveur de perspectives «expressives" centrées sur le bien-être individuel et le maintien de la paix sociale (Bernstein, 1977). Si les adultes se "serrent les coudes", ces établissements arrivent parfois à «tenir » pendant une durée plus ou moins longue tout en voyant leurs mauvais résultats se maintenir ou s'aggraver. Toutefois, si la mobilité et la mésentente sont trop fortes, ils sombrent dans l'anomie (van Zanten, 2001).
II arrive cependant que certains établissements en bas de l'échelle et ayant peu de chances d'améliorer à court terme leur attractivité choisissent de tourner le dos à la compétition non pas dans une logique de résignation, mais dans une logique au contraire de "mobilisation volontariste " en faveur du développement intellectuel et de la réussite des élèves (van Zanten et al., 2002). Les établissements qui privilégient cette posture pédagogique et éthique ont généralement des difficultés de moindre ampleur et une image moins négative que les établissements précédents. Ils se caractérisent aussi par l'existence d'un consensus moral et pragmatique entre des directeurs impliqués et charismatiques et un groupe d'enseignants motivés et dynamiques. La participation active d'une fraction des parents de classes moyennes ou des classes populaires souhaitant améliorer le capital social collectif de l'établissement au sein de l'établissement s'avère aussi déterminante (van Zanten, 2006). La capacité de ces établissements à être efficaces et à lutter contre les effets négatifs de la ségrégation dépend néanmoins aussi de l'octroi de ressources complémentaires au titre de la discrimination positive, ce qui est le cas en Belgique, en France, en Hongrie et au Portugal, mais rarement de façon suffisante. Elle dépend aussi du soutien qu'ils reçoivent de la part de l'encadrement intermédiaire, celui-ci étant présent de façon variable suivant les contextes nationaux et locaux et les réseaux d'enseignement.

\section{CONCLUSION}

Une conclusion importante de la recherche est donc que les différents domaines d'activité des établissements et leur orientation générale sont fortement influencés non seulement par les politiques éducatives nationales et locales et par des processus internes liés à l'histoire de l'établissement et aux caractéristiques de ceux qui y travaillent, mais aussi par des dynamiques locales et, plus particulièrement, par les interdépendances compétitives entre établissements. Ce résultat, obtenu par la comparaison d'une diversité de contextes nationaux et locaux, est important car il enrichit la connaissance sur le fonctionnement des établissements d'enseignement et fournit quelques pistes d'action supplémentaires par rapport aux recherches existantes pour améliorer leur efficacité et leur équité. Nous pensons notamment aux travaux sur "l'école efficace " qui ont développé des modèles qui ne peuvent pas être généralisés à l'ensemble 
des établissements parce qu'ils ont été conçus sans tenir suffisamment compte non seulement des effets de la composition sociale du public d'élèves de l'établissement (Thrupp, 1999), mais aussi des effets de la compétition avec les autres établissements sur les choix éducatifs des professionnels de l'éducation.

Par ailleurs, comme d'autres recherches, nos analyses tendent à montrer qu'il est nécessaire de limiter ces interdépendances compétitives en vue de rendre les établissements plus performants et plus égalitaires (Hardman \& Levacic, 1997, Lauder et al., 1999 ; Felouzis, 2005). Certes, la menace ou la mise en œuvre de stratégies de défection par les parents et l'« émulation compétitive » qu'elles engendrent peuvent parfois avoir des effets positifs pour des établissements situés en haut de la hiérarchie ou dans une position moyenne qui tendraient à se comporter comme des «monopoles indolents " (Hirschman, 1976). Même dans ces cas, cependant, la compétition pour les élèves et le passage d'une logique de «rente » à une logique de " conquête " semble engendrer davantage un travail de fabrication d'une bonne image vis-à-vis de l'extérieur que d'amélioration réelle de l'activité à l'intérieur (Ball, 1997). En outre, dans la très grande majorité des établissements qui accueillent un public hétérogène d'élèves et dont les résultats sont moyens ou faibles, cette compétition engendre de fortes tensions entre la nécessité de développer un ensemble de tactiques pour retenir un nombre suffisant de bons élèves et celle de prendre en charge l'accompagnement des élèves en difficulté. Or, l'arbitrage se fait souvent en faveur du premier type de contraintes car les professionnels de l'éducation y sont poussés par les parents les plus actifs et y trouvent des gratifications professionnelles plus immédiates. Enfin, il est évident que les établissements qui accueillent déjà une proportion importante d'élèves en difficulté ont tout à perdre d'une compéti- tion à laquelle ils ne peuvent participer à armes égales et dans laquelle ils risquent de perdre des élèves et des parents jouant un rôle important dans le maintien d'un fonctionnement normal.

II n'est pas évident, cependant, de chercher à limiter ces phénomènes. Ils s'ancrent en effet non seulement dans des choix politiques, mais dans des processus sociaux et culturels qu'il est difficile de modifier. II s'agit d'une part du souci d'un nombre croissant de familles des classes moyennes et supérieures de doter leurs enfants d'un environnement scolaire leur permettant d'obtenir des avantages compétitifs en matière de scolarisation et d'insertion professionnelle et de se développer à l'abri des risques académiques et sociaux associés à la fréquentation d'enfants des milieux défavorisés (van Zanten, 2002). II s'agit également de la volonté de certains établissements de garder ou étendre leurs privilèges dans des systèmes d'enseignement plus ouverts qu'autrefois et ayant de ressources limitées pour assurer la bonne scolarité de tous. Néanmoins, d'autres volets de notre recherche nous ont permis de montrer que les insuffisances des régulations politiques nationales et locales, notamment le manque de coordination entre les diverses agences publiques ou privées compétentes dans un territoire donné participent aussi du développement non planifié de la compétition. Ceci permet de supposer qu'une amélioration de l'organisation des systèmes d'enseignement qui prendrait en compte les " interdépendances compétitives " contribuerait de façon significative à limiter leurs effets les plus négatifs (Maroy, 2006).

Agnès van Zanten agnes.vanzanten@sciences-po.fr Observatoire du changement social CNRS-FNSP

\section{BIBLIOGRAPHIE}

Bagley C. ; Woods P. \& GlatTeR R. (1996). « Scanning the market: school strategies for discovering parental perspectives ". Educational Management and Administration, vol. $24, \mathrm{n}^{\circ} 2$, p. $125-138$.

BALL S. J. (1997). «Good school/bad school : paradox and fabrication ". British Journal of Sociology of Education, vol. $18, n^{\circ} 3$, p. $317-336$.

BALL S. \& VAN ZANTEN A. (1998). "Logiques de marché et éthiques contextualisées dans les systèmes scolaires français et britannique ", Éducation et sociétés, $\mathrm{n}^{\circ} 1$, p. 47-71.
Ballion R. (1991). La Bonne école : évaluation et choix du collège et du lycée. Paris : Hatier.

Bernstein B. (1977). Class, Codes and Control. III. Towards a Theory of Educational Transmissions. London: Routledge \& Kegan Paul.

Delvaux B. \& VAN ZANTEN A. (2004). Les espaces locaux d'interdépendance entre établissements: une comparaison européenne. Rapport de recherche disponible sur Internet à l'adresse: http://www.girsef.ucl.ac.be/ deliverable7.pdf (consulté le 7 novembre 2006).

EDWARDS T. \& WHITTY G. (1997). "Marketing quality : traditional and modern versions of Educational excellence ». 
In R. Glatter, P. Woods \& C. Bagley (éd.), Choice and Diversity in Schooling. London : Routledge.

FELouzis G. (2005). "Performances et "valeur ajoutée" des lycées: le marché scolaire fait des différences ". Revue française de sociologie, vol. 46, $n^{\circ} 1$, p. 3-36.

GewirTz S.; BALL S. \& Bowe R. (1995). Markets, Choice and Equity in Education. Buckingham [Mass.] : Open University Press.

HARDMAN J. \& LEVACIC R. (1997). « The impact of competition on secondary schools ». In R. Glatter, P. Woods \& C. Bagley (éd.), Choice and Diversity in Schooling. London : Routledge.

HIRSCHMAN A. (1970). Exit, Voice and Loyalty. Responses to Decline in Firms, Organizations and States. Cambridge [Mass.] : Harvard University Press, 1970.

Lauder H. ; Hughes S. ; Watson S. ; Waslander M. ; Thrupp R. ; Strathdee I.; SimiYu A. ; Dupuis J.; McGlinn J. \& HAMLIN J. (1999). Trading in Future: Why Markets in Education Don't Work. Buckingham [Mass.] : Open University Press.

MAROY C. (2006). École, régulation et marché : une analyse de six espaces scolaires en Europe. Paris : PUF [à paraître].

MAROY C. \& Dupriez V. (2000). "La régulation dans les systèmes scolaires: proposition théorique et analyse du cadre structurel en Belgique francophone ». Revue française de pédagogie, $\mathrm{n}^{\circ} 130$, p. 73-87.
MEYER J. \& ROWAN B. (1978). «The structure of Educational organizations ». In M. Meyer et al. (éd.), Environments and Organizations. San Francisco : Jossey-Bass.

THRUPP M. (1999). Schools Making a Difference: Let's Be Realistic! Buckingham [Mass.] : Open University Press.

VAN ZANTEN A. (1999). "Les chefs d'établissement et la justice des systèmes d'enseignement en France et en Angleterre ». In D. Meuret (dir.), La justice du système éducatif. Bruxelles : De Boeck.

VAN ZANTEN A. (2001). L'école de la périphérie : scolarité et ségrégation en banlieue. Paris : PUF.

VAN ZANTEN A. (2002). « Les classes moyennes et la mixité scolaire 》. Les Annales de la recherche urbaine, $n^{\circ} 93$, p. 131-140.

VAN ZANTEN A. (2006). "Les choix scolaires dans la banlieue parisienne : defection, prise de parole et évitement de la mixité ". In H. Lagrange (éd.), La cohesion sociale à l'épreuve des inégalités. Paris : PUF [à paraître].

van Zanten A. ; Grospiron M.-F. ; Kherroubi M. \& Robert A. (2002). Quand l'école se mobilise. Paris : La Dispute.

VERHOEVEN M. (1999) "Procéduralisation et réflexivité : des outils pour la régulation des établissements scolaires? ". Education et sociétés, $\mathrm{n}^{\circ} 4$, p. 143-164.

YAIR G. (1996). "School Organization and Market Ecology : A Realist Sociological Look at the Infrastructure of School Choice ». British Journal of Sociology of Education, vol. $17, \mathrm{n}^{\circ} 4$, p. 453-471. 